\title{
Satisfaction with Hearing Aids among Aged Patients with Different Degrees of Hearing Loss and Length of Daily Use
}

\author{
Rezvan Dashti ${ }^{1}$, Farzad Faraji Khiavi ${ }^{2}$, Seyyed Jalal Sameni ${ }^{3}$, and Arash Bayat ${ }^{4}$ \\ ${ }^{1}$ Musculoskeletal Rehabilitation Center, School of Rehabilitation, Ahvaz Jundishapur University of Medical Sciences, Ahvaz, \\ ${ }^{2}$ Department of Health Services Administration, School of Health, Ahvaz Jundishapur University of Medical Sciences, Ahvaz, \\ ${ }^{3}$ Department of Audiology, School of Rehabilitation, IUMS, Lecturer, Iran Medical Sciences University, Tehran, \\ ${ }^{4}$ Hearing and Speech Research Center, Department of Audiology, School of Rehabilitation, Ahvaz Jundishapur University of Medical \\ Sciences, Ahvaz, Iran
}

\begin{abstract}
Received October 16, 2014
Revised December 9, 2014

Accepted March 26, 2015
\end{abstract}

Background and Objectives: The evaluation of subjective benefits and positive effects of hearing aids in daily is important for measuring the treatment outcome. The aim of this project was to investigate the degree of satisfaction of aged users with their hearing aids using the Satisfaction with Amplification in Daily Life (SADL) scale, which emphasizes non-auditory factors contributing to satisfaction as well as benefit. Subjects and Methods: The Persian version of SADL scale was completed by 40 patients who received monaural hearing aid fitting at south of the Iran from December 2013 and March 2014. SADL subscales of the SADL were evaluated according to the type and degree of hearing loss, the pure tone audiogram pattern and shape and type of the hearing aid. Results: The results associated with the SADL subscales revealed a greater satisfaction associated with the Positive Effect and Service and Costs subscales. Subjects with different degree of hearing loss were very satisfied in terms of positive effect subscale. Conclusions: Participants reported a considerable level of satisfaction with their hearing aids. Appropriate guidance for using hearing aids and spending more time for counseling can improve the satisfaction level of this age group.

J Audiol Otol 2015;19(1):14-19

\section{Introduction}

As age increases, the importance of health promoting behaviors become more prominent owing to their increasing contribution to maintaining the functions required for living independently. The utmost issues concerning the quality of life for the elderly have to do with ensuring their adequate independence in physical and cognitive activities for carrying on living effectively. Aggravation of poor health and chronic diseases as age goes up brings about constraints upon physical activities of the elderly [1].

Hearing loss (HL) has been reported as one of the most prevalent chronic diseases among the elderly [2,3]. Currently,

This is an Open Access article distributed under the terms of the Creative Commons Attribution Non-Commercial License (http://creativecommons. org/licenses/by-nc/3.0/) which permits unrestricted non-commercial use, distribution, and reproduction in any medium, provided the original work is properly cited.
$15 \%$ of people older than 15 years of age, or 600 million people, have some degree of a hearing impairment (HI), whilst 5.4\% of those have a disabling HL. However, in developed countries, only $2 \%$ of the population aged $20-24$ suffer from HL this percentage increases to $85 \%$ in people aged $75-84$. An epidemiology of hearing loss study recently reported an increased prevalence of measured hearing loss of $21 \%$ for people aged $48-59$ and $90 \%$ for those aged more than 80 [5].

Although there is no accurate information about prevalence of hearing problems in Iran, some research in Ardabil state showed that 7.1 per thousand individuals suffered HIs and 4.3 per thousand were deaf. The mentioned research estimated hearing problems prevalence 82.7 and 67.3 per thousand respectively for urban and rural elderly [6]. Based on some predictions, Iran population will contain more than 10 million elderly in 2020 . So elderly hearing problems need to be considered in multiple aspects including satisfaction with 
hearing aid [6].

In recent years, $\mathrm{HL}$ in the elderly has not been dealt with from a biological aspect solely; rather, the social and individual impacts of the impairment upon communication with others and socialization as well as the economic aspects are also taken into account [7]. Among all sensory impairments potentially affecting the elderly, communicative impairment due to $\mathrm{HL}$ is one of the most frustrating and harmful major impairments, causes a reduction in a person's quality of life $[8,9]$. This is mainly because hearing not only affects the elderly, but also it affects people in their company, i.e. the family and colleagues, and they have to speak to the elderly more loudly and clearly [10]. As a consequence, this type of HL due to ageing (presbyacusis) may causes an alarm about potential depression, isolation, inattention, decreased self-confidence, diminished social roles, increased dependence on others and poor performance as well as cognitive impairments in the elderly [2,8-14], particularly those who have not received any therapeutic intervention for their HL [12].

Presbyacusis can be treated through neither medication nor a surgical operation $[2,15]$. The only effective solution is to amplify sounds by hearing aids [2]. Suitably adjusted hearing aids are often used for rehabilitation of the elderly, a method that has proven positively effective on the well-being of the elderly and improvement of their daily lives [13,16-18]. In other words, failure in diagnosis and timely treatment of HIs reduces the quality of life and ability of the elderly [13] .

While objective outcome-measurement methods, such as speech recognition scores, measure the benefit provided by the hearing aid in the clinical settings, but they may not be accurate indicators of hearing aid performance in the real world. In recent years, a number of self-reporting questionnaires have been developed to evaluate the entire process of hearingaid intervention in daily living situations. Most of these measurements rely on the global satisfaction with amplification solely, but Cox and Alexander, who developed Satisfaction Amplification in Daily Life (SADL) questionnaire, reported one global score can't determine why patients may satisfied with their hearing aid and can't recommend approaches for improve or remedy disability in rehabilitation process. The SADL has been determined as a valid and reliable method to assess multiple dimensions of satisfaction with hearing aids in real life situations.

Hosford-Dunn and Halpern [19] suggested that SADL could be regard as a gold standard for measuring amplification outcome. Uriarte, et al. [20], also used SADL for measuring satisfaction of the elderly fitted with hearing aids and reported a high level of satisfaction following amplification in the studied population.
Satisfaction with hearing aids may affected by several factors, including the age of the patient, the cost of the hearing aids, the amount of experience with amplification, degrees of $\mathrm{HL}$ and how many hours patients use their hearing aids per day [20]. Although these variables can have an impact on hearing aid satisfaction, the importance of them to patient scores has not been fully understood. The aim of this study was to investigate the degree of satisfaction of aged users with their hearing aids using the SADL scale, which emphasizes non-auditory factors contributing to satisfaction as well as benefit as well as to determine whether the level of satisfaction may influenced by various degrees of $\mathrm{HL}$ and lengths of daily use or not?

\section{Subjects and Methods}

\section{Subjects}

A total of 40 subjects (30 males, 10 females) were recruited in our Hearing Aid Clinic and participated in the study; the mean age was 74.62 years and the age range was 66 to 86 years. All the data were collected between December 2013 and March 2014 from South of Iran (Kangan City). All participants enrolled in the study met the following selection criteria: 1) HL that was flat or gently sloping (from 250 to 4000 $\mathrm{Hz})$; 2) no known medical or surgically treatable ear disease; 3) no known fluctuating or progressing hearing loss; and 4) wearing the hearing aids for at least six months, this period is suitable for adapting to hearing aids.

The hearing aid fittings were monaural in all cases, and they were fitted with three different technologies: digital $(n=24)$, analogue $(\mathrm{n}=10)$, and digitrim $(\mathrm{n}=6)$.

\section{Procedure}

The satisfaction of the elderly with use of hearing aids in daily life was evaluated through the Persian version of SADL questionnaire. In order to measuring patients' satisfaction with hearing aids, standard Persian version of SADL questionnaire was used. The questionnaire was validated through face and content validity by 5 experts. Cronbach's alpha calculated 0.80 for reliability of data gathering instrument. Subjects were asked to fill out the questionnaire individually, or through direct interview. Due to some subjects' low literacy, the questions of questionnaire were read aloud and completed by a trained interviewer.

The SADL is a 15 -item questionnaire that measures the subjective satisfaction of hearing aids. It provides a global score indicating overall satisfaction and profile of four subscale scores: 1) "Positive Effect" which consists of items on improved functioning in psychoacoustic and psychological 
realms, 2) "Negative Features" that address annoying aspects of hearing aid use such as performance in noisy situations, presence of feedback and use of telephone, 3) "Service and Cost", which devoted to hearing rehabilitation services and amplification costs and, 4) "Personal Image", that covers aspects of self-image and hearing aid stigma. The questions were answered with a 7-point scale including "a) not at all, b) a little, c) somewhat, d) medium, e) considerably, f) greatly, and g) tremendously. Each answer is scored with 1 to 7 points. For 11 questions 'g' meant "tremendously" and showed maximum satisfaction thus was assigned a score of 7. But 'a' meant "not at all" and demonstrated maximum dissatisfaction and thus was given 1 score. For the other 4 questions, the score was inverted; meaning that "tremendously" meant complete dissatisfaction and was scored 1, whereas "not at all" indicated completely satisfied and scored 7 . The overall scores of the four subscales were calculated according to the average value of the scores attained from each component item.

The study plan was approved by the Medical Ethics Committee of the Ahvaz Jundishapur University of Medical Sciences, Ahvaz, Iran. The participants provided written informed consent for the study.

\section{Statistical analysis}

Statistical analyses were performed with SPSS software (SPSS Inc., Chicago, IL, USA). We used chi-square test to observe any correlations between satisfaction with hearing aid and length of hearing aid use. Analysis of variance was used to compare global and subscale SADL scores according to time of daily use. The level of significance was set at $p<0.05$.

\section{Results}

In terms of education, $36(90 \%)$ were illiterate, $3(7.5 \%)$ finished secondary school and $1(2.5 \%)$ had high school diploma. Twenty-nine participants $(86.25 \%)$ suffered from sensorineural hearing loss; the remaining 11 (13.15\%) showed mixed hearing loss.

According to Table 1 the most of subjects had moderate to severe hearing loss and the minimum percent of users had moderate hearing loss.

Table1. Distribution of patients according to the degree of hearing loss

\begin{tabular}{lcccccc}
\hline \multirow{2}{*}{ Degree of hearing loss } & \multicolumn{3}{c}{ Right ear } & & \multicolumn{3}{c}{ Left ear } \\
\cline { 2 - 3 } \cline { 6 - 7 } & $n$ & $\%$ & & $n$ & $\%$ \\
\hline Moderate & 9 & 22.5 & & 5 & 12.5 \\
Moderate to severe & 20 & 50 & & 21 & 52.5 \\
Severe & 11 & 27.5 & & 14 & 35 \\
\hline
\end{tabular}

As it is shown in the Table 2, most of the subjects use hearing aids more than 8 hours a day and small number use them less than 4 hours a day.

In order to assess the correlation between age and level of satisfaction, elderly users were divided into 2 age groups; i.e., $65-74(n=20)$ and $75-90$ years $(n=20)$. There were 20 subjects in each group.

The levels of satisfaction of each SADL subscale are presented in Fig. 1. Users were categorized as "very satisfied", "satisfied", and "dissatisfied" in each SADL subscale, according to their global score.

In in terms of cost and service $14(35 \%)$ subjects were very satisfied, $20(50 \%)$ satisfied, and $6(15 \%)$ were dissatisfied. As for personal image, however, $1(2.5 \%)$ of the subjects was very satisfied, $31(77.5 \%)$ satisfied, and $8(20 \%)$ were dissatisfied. Seventeen $(42.5 \%)$ of the subjects were satisfied and 23 $(57.5 \%)$ were dissatisfied in terms of Negative Feature scale.

In regards of the positive effects, $29(72.5 \%)$ of the subjects were very satisfied, 10 (25\%) satisfied, and 1 (2.5\%) was dis-

Table 2. Distribution of patients based on the length of time of daily use, lifetime of hearing aid experience with current hearing aid variables

\begin{tabular}{lrc}
\hline Characteristic & $\mathrm{n}$ & $\%$ \\
\hline Lifetime hearing aid experience & & \\
Less than 6 weeks & 26 & 65 \\
6 weeks to 11 months & 1 & 2.5 \\
1 to 10 years & 8 & 20 \\
Over 10 years & 5 & 12.5 \\
Daily hearing aid use & 4 & \\
1 to 4 hours & 7 & 17.5 \\
4 to 8 hours & 29 & 72.5 \\
8 to 16 hours & & \\
Experience with current hearing aid & 5 & 12.5 \\
6 weeks to 11 months & 31 & 77.5 \\
1 to 10 years & 4 & 10 \\
Over 10 years
\end{tabular}

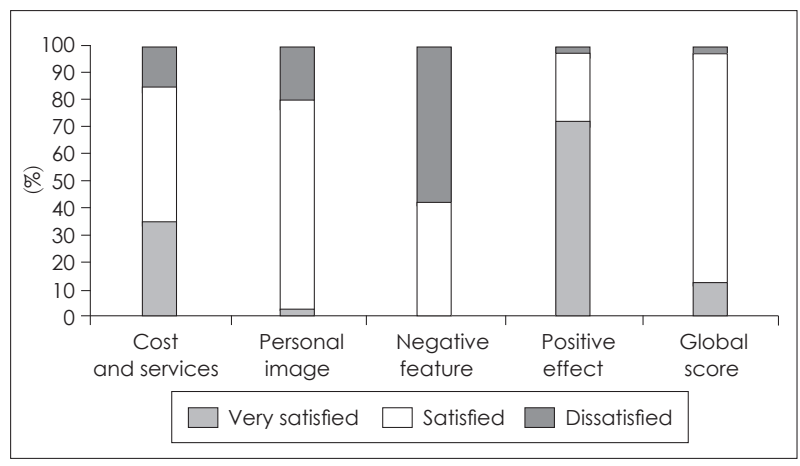

Fig. 1. Percentage of very satisfied, satisfied and dissatisfied users in each subscale and satisfaction global scores in the SADL scale. SADL: Satisfaction Amplification in Daily Life. 
Table 3. Satisfaction with hearing aids based on time of daily use

\begin{tabular}{lcccc}
\hline \multirow{2}{*}{ SADL subscale } & \multicolumn{3}{c}{ Time of daily use of hearing aid } & \multicolumn{1}{c}{$p$-value } \\
\cline { 2 - 4 } & 1 to 4 hours & 4 to 8 hours & 8 to 16 hours & 0.34 \\
Cost and services & $4.08 \pm 1.25$ & $5.09 \pm 1.30$ & $4.87 \pm 1.07$ & 0.06 \\
Personal image & $3.66 \pm 0.94$ & $4.33 \pm 0.69$ & $4.59 \pm 0.70$ & 0.78 \\
Negative features & $3.69 \pm 0.90$ & $3.56 \pm 0.54$ & $3.00 \pm 0.55$ & 0.07 \\
Positive effect & $3.83 \pm 1.03$ & $3.52 \pm 1.13$ & $3.52 \pm 0.71$ & 0.03 \\
Global score* & $4.91 \pm 0.50$ & $6.02 \pm 0.44$ & $5.94 \pm 0.93$ & \\
\hline
\end{tabular}

$* p<0.05$. SADL: Satisfaction Amplification in Daily Life

Table 4. Satisfaction with hearing aids based on degree of hearing loss

\begin{tabular}{lcccc}
\hline \multirow{2}{*}{ SADL subscale } & \multicolumn{3}{c}{ Degree of hearing loss } & P-value \\
\cline { 2 - 4 } & Moderate & Moderate to severe & Severe & 0.10 \\
Cost and services & $5.10 \pm 1.15$ & $4.85 \pm 1.1$ & $3.86 \pm 0.69$ & 0.88 \\
Personal image & $4.52 \pm 0.72$ & $4.43 \pm 0.79$ & $4.33 \pm 0.88$ & 0.01 \\
Negative features* & $3.10 \pm 0.76$ & $3.82 \pm 0.60$ & $4.00 \pm 1.05$ & 0.35 \\
Positive effect & $5.98 \pm 0.78$ & $5.88 \pm 0.80$ & $5.33 \pm 1.38$ & 0.30 \\
Global score & $4.94 \pm 0.57$ & $4.97 \pm 0.44$ & $4.57 \pm 0.60$ & \\
\hline
\end{tabular}

* $p<0.05$. SADL: Satisfaction Amplification in Daily Life

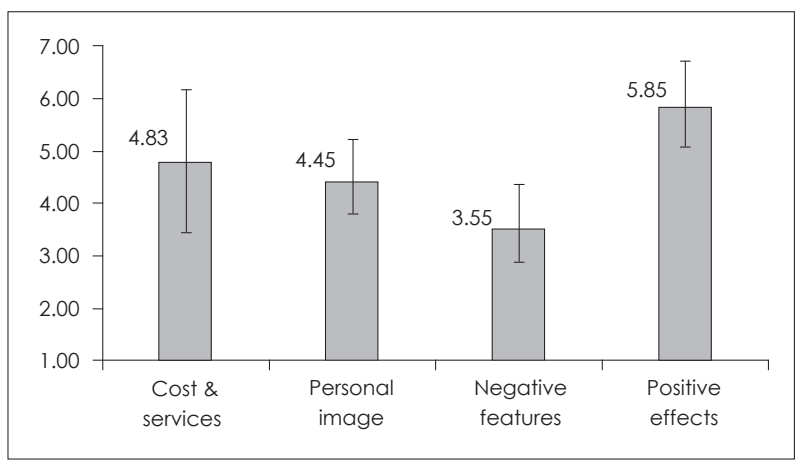

Fig. 2. Mean subscale scores of SADL. SADL: Satisfaction Amplification in Daily Life.

satisfied with their hearing aids. In terms of the global score 5 $(12.5 \%)$ of the users were very satisfied, $34(85 \%)$ satisfied and $1(2.5 \%)$ was dissatisfied.

We found a significant difference between the length of time of daily use of hearing aids and the satisfaction level of elderly which was more remarkable for SADL global score (Table 3).

As demonstrated in Table 4, subjects with different degree of hearing loss were very satisfied in terms of positive effect subscale. Accordingly, the mean score of personal image subscale shows moderate satisfaction of older adults. The minimum level of satisfaction resulted in negative feature subscale (Fig. 2).

As demonstrated in Fig. 2, the highest level of satisfaction is seen in positive effect subscale, while the subjects are the least satisfied in terms of negative features. The satisfaction levels in others subscales (Cost and Services, and Personal Image) for all users were assessed as fairly satisfied. In terms of sub- ject global scores, 5 (12.5\%) subjects are very satisfied, 34 $(85 \%)$ satisfied, and only $1(2.5 \%)$ was dissatisfied.

\section{Discussion}

Satisfaction with hearing aids in elderly people suffering from HL has been the topic of research in many studies.

In this study the dominant number the subjects were male. The average final score in global SADL was $4.91 \pm 0.52$ which showed that users were moderately satisfied with their hearing aids. Compared to similar studies, in this study we found lower level of satisfaction while in a study by Cox and Alexander [21] it was reported that the users had a considerably high satisfaction and it is the same with the result reached in Viega, et al. [22] and de Carvalho [23].

According to the significant difference between degree of $\mathrm{HL}$ and satisfaction level with hearing aids, as HL increased, the cost of hearing aids increased too.

The average of individual scoring on positive effect subscale found in the current study was higher than subscales of SADL, that is $(5.88 \pm 0.88)$ which shows the users' great satisfaction with their hearing aids in the community they live in. As for the sound quality of hearing aids, only a few subjects expressed their dissatisfaction with acoustic features and psychological impact of their hearing aids. These results were in compliance with the original research conducted by Cox and Alexander [24].

The mean score of the negative feature subscale was lower than the ones found in this study which is $(3.55 \pm 0.80)$. One of the main complaints of users is having difficulty in talking 
on the phone; indeed, it is in telephone use that the technological limitation of hearing aids are revealed [19]. However, the results in this regard are similar to other studies; for instance Carvelho reported this and gained the lowest satisfaction in this subscale [21]; and so did Hosford-Dunn and Halpern [19]. Because of the phone is one of the communication method, the audiologist should pay attention to this point in adjustments.

The mean scale in personal image subscale was high which shows users' satisfaction. Personal image subscale is related to the users' self-image and hearing aid stigma. In Cox and Alexander research the highest level of satisfaction was seen in this sub scale. It showed that patients usually did not associate wearing hearing aids with disability. This result matched the result of the current study; according to the mean score of personal image subscale and the satisfaction of older adults in this study, the authors found that stigma of HL which is caused by the use of hearing aids was the least important factor for the users. They accepted their limitation without having the perception that HL has prevented them from having a normal life.

The average score of cost $\&$ services subscale was $4.83 \pm$ 1.13 which showed moderately high satisfaction of users. But one of the factors that reduced this satisfaction was the high cost of hearing aids particularly the modern ones, that is digital hearing aids. The cause might be attributed to the inadequate coverage of insurance organizations to pay for hearing aids cost as a therapeutic prosthesis.

All of the subjects were selected from the elderly people and thus because of their age they were more likely to face accommodation problems with their hearing aids. $72.5 \%$ of them were reported using hearing aids for above 8 hours per day which means that most of the day time the subjects use amplification devices to communicate with others. In this situation, satisfaction with hearing aids can improve communication with others and give them more opportunities to participate in social activities. It reduces stress and anxiety among the users and promotes self-confidence in them. The audiologist and aural rehabilitation teams should attend to psychosocial health status of the clients when planning rehabilitation programs.

The SADL questionnaire was used successfully in several countries. This questionnaire is considered a significant tool in verification steps, actually the patients' viewpoints are playing some key roles in efficacy of hearing aid. It seems the audiologists should focus on counseling them and create realistic expectations. Users' responses to this self-assessment can be related to personal conception and opinions, therefore the SADL may considered a good measurement for showing the satisfaction level of users after fitting hearing aid [19]. Satisfaction is an objective concept and determination of satis- faction is important in auditory rehabilitation not only because of assaying how individuals reply to intervention but also because of showing improvements that include interpersonal interaction between patients and audiologist, availability of services, continuity of services and financial concerns [20].

In general, the population participated in this study was satisfied with their hearing aids during their daily life regardless of the type of their HL and degree of HL. There was a high level of satisfaction with hearing aids in all subscales of SADL. Appropriate counseling and guidance can be one of the important factors of satisfaction.

\section{Conclusion}

In general, the population participated in this study was satisfied with their hearing aids during their daily life regardless of the type of their hearing loss and degree of hearing loss. There was a high level of satisfaction with hearing aids in all subscales of SADL. Appropriate counselling and guidance can be one of the important factors of satisfaction.

\section{Acknowledgments}

The source of data used in this paper was from MSc thesis of Rezvan Dashti, student of Ahvaz Jundishapur University of Medical Sciences; and financial support was provided by Ahvaz Jundishapur University of Medical Sciences (Master Thesis grant no: pht9305).

\section{REFERENCES}

1) Lee TW, Ko IS, Lee KJ. Health promotion behaviors and quality of life among community-dwelling elderly in Korea: a cross-sectional survey. Int J Nurs Stud 2006;43:293-300.

2) Lotfi Y, Mehrkian S, Moossavi A, Faghih-Zadeh S. Quality of life improvement in hearing-impaired elderly people after wearing a hearing aid. Arch Iran Med 2009;12:365-70.

3) Dalton DS, Cruickshanks KJ, Klein BE, Klein R, Wiley TL, Nondahl DM. The impact of hearing loss on quality of life in older adults. Gerontologist 2003;43:661-8.

4) World Health Organization. WHO global estimates on prevalence of hearing loss. Geneva: WHO;2012. Retrieved from http://www.who. int/pbd/deafness/WHO_GE_HL.pdf.

5) Al-Ruwali N, Hagr A. Prevalence of presbyacusis in the elderly Saudi Arabian population. J T U Med Sc 2010;5:21-6.

6) Hajloo N, Ansari S. Prevalence and causes of hearing handicap in Ardabil province, Western Iran. Audiol 2011;20:116-27.

7) Angeli RD, Jotz GP, de Barba MC, Demeneghi PGM, de Mello CHP. [Effectiveness of a program of auditory prothetization in elders through the application of HHIE-S Questionnaire]. Intl Arch Otorhinolaryngol 2009;13:277-80.

8) Kelly RJ, Atcherson SR. Quality of life for individuals with hearing impairment who have not consulted for services and their significant others: same- and different-sex couples. J Commun Disord 2011;44:336-44.

9) Calviti KC, Pereira LD. Sensitivity, specificity and predictive values of hearing loss to different audiometric mean values. Braz J Otorhinolaryngol 2009;75:794-800.

10) Wingfield A, Peelle JE. How does hearing loss affect the brain? Aging Health 2012;8:107-9. 
11) Perez E, Edmonds BA. A systematic review of studies measuring and reporting hearing aid usage in older adults since 1999: a descriptive summary of measurement tools. PLoS One 2012;7:e31831.

12) Ciorba A, Bianchini $C$, Pelucchi S, Pastore A. The impact of hearing loss on the quality of life of elderly adults. Clin Interv Aging 2012;7:159-63.

13) Gates GA, Murphy M, Rees TS, Fraher A. Screening for handicapping hearing loss in the elderly. J Fam Pract 2003;52:56-62.

14) Arlinger S. Negative consequences of uncorrected hearing loss--a review. Int J Audiol 2003;42 Suppl 2:2S17-20.

15) Chang WH, Tseng HC, Chao TK, Hsu CJ, Liu TC. Measurement of hearing aid outcome in the elderly: comparison between young and old elderly. Otolaryngol Head Neck Surg 2008;138:730-4.

16) Gopinath B, Schneider J, Hartley D, Teber E, McMahon CM, Leeder SR, et al. Incidence and predictors of hearing aid use and ownership among older adults with hearing loss. Ann Epidemiol 2011;21: 497-506.

17) Hidalgo JL, Gras CB, Lapeira JM, Martínez IP, Verdejo MA, Rabadán FE, et al. The Hearing-Dependent Daily Activities Scale to evaluate impact of hearing loss in older people. Ann Fam Med 2008;6:441-7.
18) Appollonio I, Carabellese C, Frattola L, Trabucchi M. Effects of sensory aids on the quality of life and mortality of elderly people: a multivariate analysis. Age Ageing 1996;25:89-96.

19) Hosford-Dunn H, Halpern J. Clinical application of the satisfaction with amplification in daily life scale in private practice I: statistical, content, and factorial validity. J Am Acad Audiol 2000;11:523-39.

20) Uriarte M, Denzin L, Dunstan A, Sellars J, Hickson L. Measuring hearing aid outcomes using the Satisfaction with Amplification in Daily Life (SADL) questionnaire: Australian data. J Am Acad Audiol 2005;16:383-402.

21) Cox RM, Alexander GC. Measuring Satisfaction with Amplification in Daily Life: the SADL scale. Ear Hear 1999;20:306-20.

22) Veiga LR, Merlo AR, Mengue SS. Satisfaction level with hearing aid in the daily life of Army Healthcare System users. Braz J Otorhinolaryngol 2005;71:67-73.

23) de Carvalho JSA. [Satisfaction of the elderly With hearing aid provided in Tocantins State-Brazil]. Intl Arch Otorhinolaryngol 2007; 11:416-26.

24) Cox RM, Alexander GC. Validation of the SADL questionnaire. Ear Hear 2001;22:151-60. 